\title{
Evaluating FDDI on the basis of Throughput and Response time
}

\author{
Farminder Singh \\ Department of Computer Science \\ Punjabi University, Patiala
}

\author{
Amandeep Singh Dhaliwal \\ Department of Computer Science \\ Punjabi University, Patiala
}

\begin{abstract}
FDDI is an optical standard for data transmission which gave a transmission speed of $100 \mathrm{Mbit} / \mathrm{s}$ in a local area network. The throughput and response time is a measure of performance of a network. The performance of the FDDI system depend on various parameters namely ring latency, TTRT, number of stations, bandwidth, transmission delay, propagation time and group velocity. The throughput or the efficiency of the network can be increased by controlling and then minimizing the ring latency with respect to TTRT. The response time can be improved by decreasing the transmission delay and propagation time with respect to the bandwidth and group velocity respectively.
\end{abstract}

\section{Keywords: TTRT, FDDI}

\section{INTRODUCTION}

Fiber distributed data interface (FDDI) is an optical standard for data transmission in a local area network (LAN). The logical topology is a ring based token network. FDDI, as a product of American National Standards Institute X3T9.5 (now X3T12), conforms to the open systems interconnection (OSI) model of functional layering of LANs using other protocols [3]. In LAN, FDDI support thousands of users. It also support real-time allocation of network bandwidth and allows the use of wide array of different types of traffic. A FDDI network has two data transmission path. One is the primary path and other is secondary path. So it is called dual ring network [5]. Due to some problem if primary ring fails then secondary ring is used for data transmission. Primary ring has the capacity of $100 \mathrm{Mbits} / \mathrm{s}$ carrying data but it extends up to $200 \mathrm{Mbits} / \mathrm{s}$ if the secondary ring is not in use. The frame size of FDDI is also larger than standard Ethernet, allowing better throughput. The FDDI is derived from the IEEE802.4 token bus timed token protocol. FDDI can be implemented as a higher speed version of a token ring and is designed to work over fiber. The basic principle of the token ring is that access of communication ring is limited to one modem at a time. Message from only a single modem is transmitted at a time. While one modem transmit its message other modems remain idle. Then, once the message travels completely around the ring, the data from the ring is removed by the modem and control (the token) is passed to the next modem. This network uses optical fiber as a transmission medium. The fiber is made up of glass which is cheaper than other transmission mediums used like coaxial cables etc.

\section{BACKGROUND}

There are number of researchers worked on FDDI to improve the efficiency and response time of the network. The demand for larger networks increased day by day which provide higher capacity and additional services. FDDI as a new LAN generation was widely accepted. $100 \mathrm{Mbit} / \mathrm{s}$ of data rate is provided by FDDI which make it suitable for connecting super computers and powerful workstations. This also supports new, more demanding applications. Although FDDI is still going through standardization, many products were becoming available on the market today. In order to achieve reliable, high performance and cost effective communication systems the problem of product design and installing strategies for FDDI networks become more important [3].

The non-timed-token protocol and timed-token protocol were two classifications of token protocols (TTPs). The queuing model was included by non-TTP and for a long time mathematical analyses have been studied and from these analyses significant results have been obtained. The TTP was studied that how the developments were took place in fiber distributed data interface (FDDI) in MAN [2]

This FDDI network use two counter rotating optical rings. In this study R Radhakrishna Pulai was considered various possible faults (like lost token, link failures, etc.). Using parameters queue length and average delay, simulation was done and the performance improvement as compared to existing fault detection and ring recovery was calculated. They also suggest some modifications to make the network more reliable. It was shown that, even when some link failure occurs, full connectivity was maintained among the stations. For link reconfiguration a distributed algorithm was also proposed in modified FDDI network when many successive as well as simultaneous link failures occur. Through simulation the performance of modified FDDI network was studied for link failure and compared with existing FDDI network [7].

FDDI token ring network provides a guaranteed throughput for synchronous message and a bounded medium access delay for each node/station was suggested by Qin Zheng [6]. He said that many real-time applications that need the timely delivery of each critical message were not effectively support by this fact. The main reason behind this was that the FDDI make sure that a medium access delay bound to nodes. The message-delivery delays may go beyond the medium-access delay bound even if a node transmits synchronous messages at a rate not larger than the guaranteed throughput. He solved this problem by developing a Synchronous Bandwidth Allocation (SBA) scheme which calculated the synchronous bandwidth necessary for each application to satisfy its message-delivery delay requirement. The results obtained were necessary for effective use of the FDDI token ring networks in supporting such real-time communications as digital video/audio transmissions and distributed control/monitoring.

The demand for larger networks increased day by day which provide higher capacity and additional services. FDDI as a new LAN generation was widely accepted. $100 \mathrm{Mbit} / \mathrm{s}$ of data rate is provided by FDDI which make it suitable for connecting super computers and powerful workstations. This also supports new, more demanding applications. Although 
FDDI is still going through standardization, many products were becoming available on the market today. In order to achieve reliable, high performance and cost effective communication systems the problem of product design and installing strategies for FDDI networks become more important. Both the basic concepts implemented in the protocol and the practical use of FDDI was the topics of this contribution by Peter David's et. al. [8].

The description of time token access method used by FDDI stations is given by Raj Jain [1]. It was emphasized that the parameter TTRT is one of the major parameter that affect the performance of a network. This parameter can be controlled by network manager to optimize the performance. Apart from TTRT, other parameters that affected the FDDI performance were the length of cable, number of active station, frame size, bandwidth, group velocity, transmission delay and propagation delay.

\section{PARAMETERS}

The parameters that are used to measure the performance of FDDI in the present study are throughput and response time.

\subsection{Throughput}

The throughput of any network is defined as the rate at which one node is transferring the data to another node in the network. The efficiency and maximum access delay (MAD) can be calculated by observing the working of space time diagram discussed here [1]. Which are used to analyze the performance of FDDI network. First a ring with three active stations is considered, which are shown in figure 4.1. After this general case will be considered with $n$ active stations. The space-time diagram is shown by the figure which represents various events on the ring. Horizontally the space is shown and vertically the time is shown. The thick horizontal line shows the token.

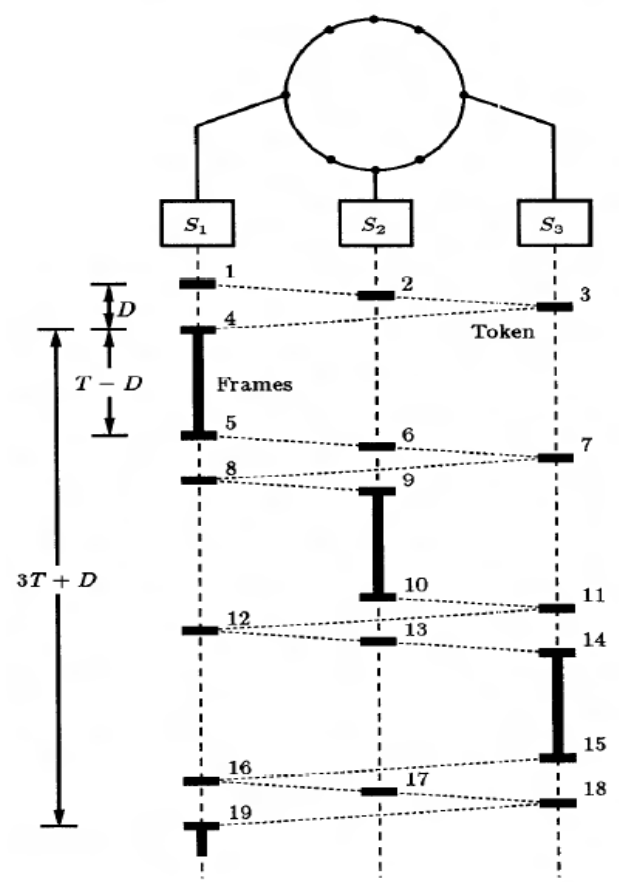

Fig 1: Space-time diagram with three active stations on an FDDI network. [1].
During each cycle, the maximum access delay for each station will be defined. Each station waits for an interval of $2 \mathrm{~T}+2 \mathrm{D}$ after releasing the token. When the load is lower, the access delay will also be lower. Therefore, for a ring with three active stations, the efficiency and access delay for each station is [1]:

$$
\begin{aligned}
& \text { Efficiency: } \eta=\frac{3(T-D)}{3 T+D} \\
& M A D=(3-1) T+2 D
\end{aligned}
$$

The above equation can be generalized to $n$ active stations by replacing 3 by $n$.

$$
\begin{aligned}
& \text { Efficiency: } \eta=\frac{n(T-D)}{n T+D} \\
& M A D=(n-1) T+2 D
\end{aligned}
$$

Equations (i) and (ii) constitute the analytical model, these equations can be used to compute the Maximum access delay and Efficiency of FDDI systems.

\subsection{Response Time}

The response time of a data network is the time between the arrival of the frame and the completion of its transmission.

In order to compute the response time, the parameters namely propagation time and transmission delay are considered in the present study.

The propagation delay is given by equation (iii) as

$$
\operatorname{Pr}=d / c
$$

Where: $\operatorname{Pr}=$ Propagation Time

$$
\begin{aligned}
& d=\text { Distance } \\
& c=\text { Group velocity }
\end{aligned}
$$

The transmission delay is given by equation (iv):

$$
T d=S / B
$$

Where: $T d=$ Transmission Delay

$$
\begin{aligned}
& S=\text { Number of bits Transmitted } \\
& B=\text { Data Rate (Bandwidth) }
\end{aligned}
$$

Equation (iii) and (iv) constitute the analytical model, the equations can be used to compute the response time of the FDDI system.

\section{RESULTS}

From the above discussion some results are calculated which are shows below by using the values given in the below tables. 
Table 1: For Efficiency

\begin{tabular}{|c|r|c|c|}
\hline Scenario & $\begin{array}{c}\text { Ring Latency } \\
(\mathrm{ms})\end{array}$ & TTRT( ms) & Stations (num) \\
\hline 1 & 10 & 165 & 100 \\
\hline 2 & 50 & 165 & 100 \\
\hline 3 & 100 & 165 & 100 \\
\hline 4 & 150 & 165 & 100 \\
\hline
\end{tabular}

\section{Efficiency vs Ring Latency}

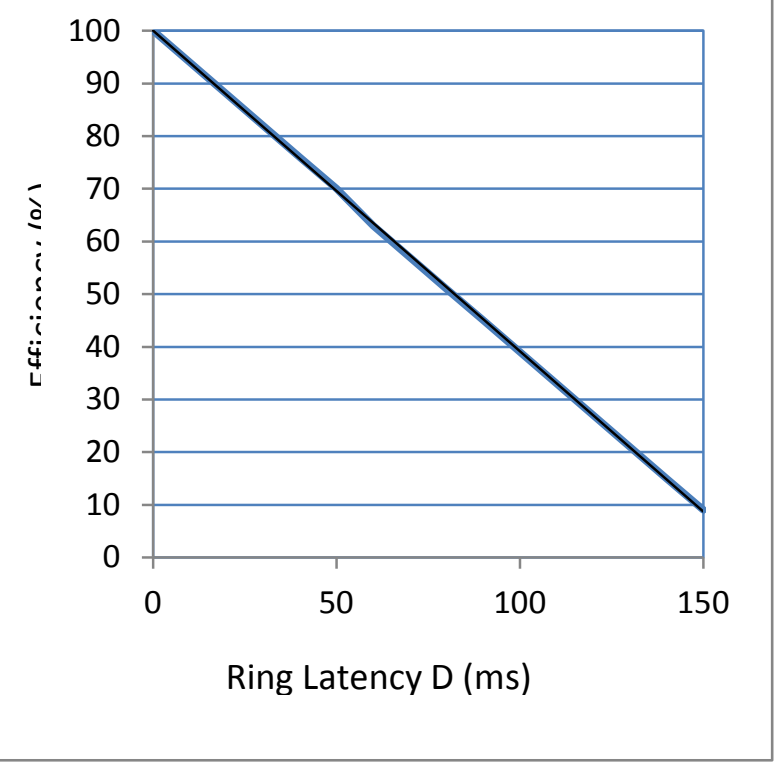

Fig 2 : Ring latency Vs Efficiency

This graph show that as the ring latency of network increases from $10 \mathrm{~ms}$ to $150 \mathrm{~ms}$ the efficiency of network decreases. When ring latency value reaches 150 the efficiency of network decreases to 0 .

Table 2: For Group velocity Vs Propagation Time

\begin{tabular}{|c|c|c|c|}
\hline Scenario & Group Velocity & Distance (in meters) & Propagation Time \\
\hline 5 & 10 & 100 & 100 \\
\hline
\end{tabular}

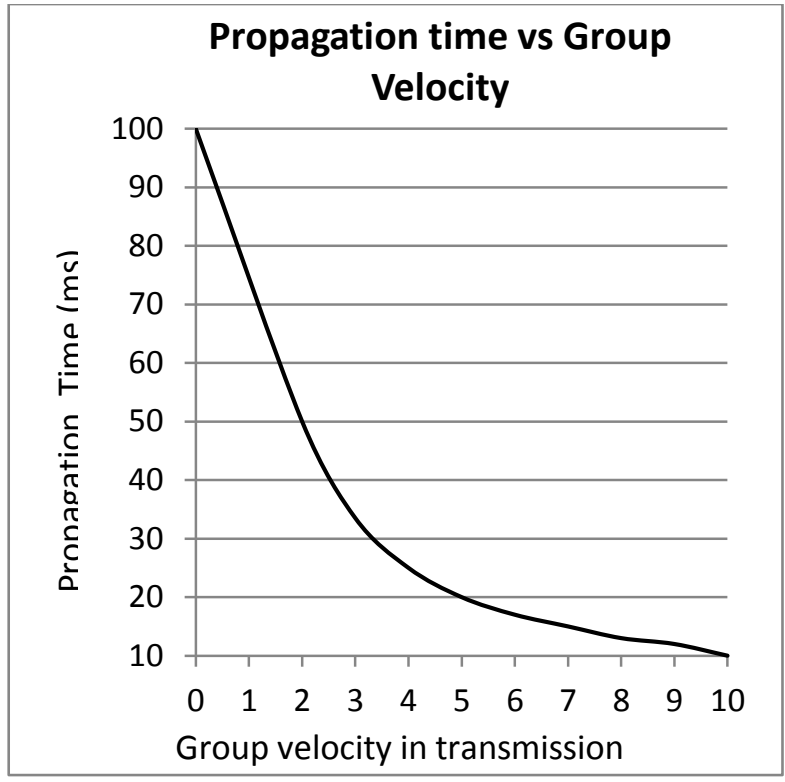

Fig 3 : Group velocity Vs Propagation Time

Above graph shows that as the group velocity increases the propagation time decreases. As the Propagation time decreased the response time of network increases.

Table 3 : For Bandwidth Vs Transmission Delay

\begin{tabular}{|c|c|c|c|}
\hline Scenario & Bandwidth & Number of Bits Transmitted (bytes) & Distance (km) \\
\hline 6 & 50 & 1000 & 100 \\
\hline
\end{tabular}

Below graph shows that as the bandwidth of network increased the transmission delay decreased and when transmission delay decreased the response time of network increased.

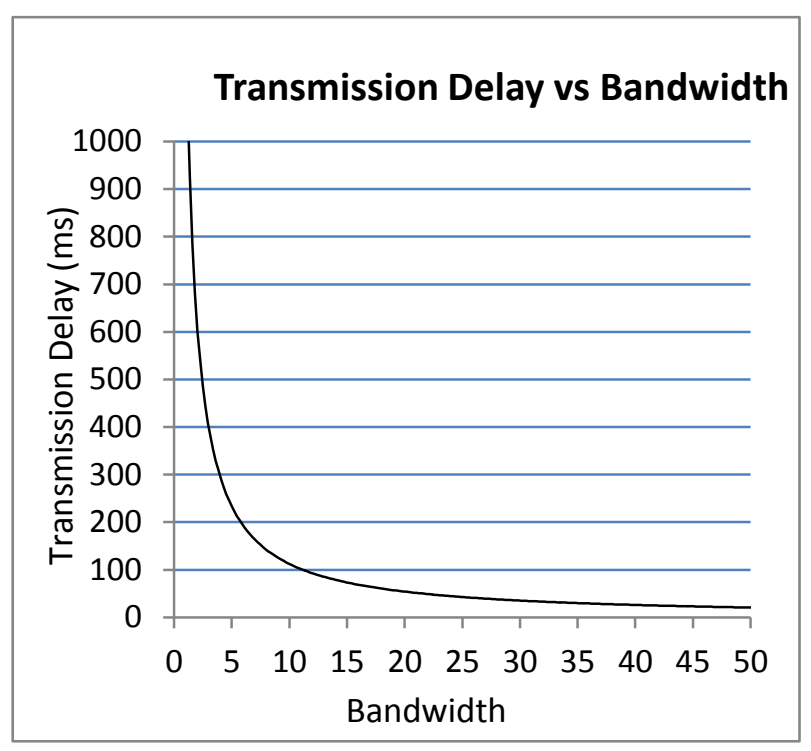

Fig 4 : Bandwidth Vs Transmission Delay 


\section{CONCLUSION}

In this paper we have evaluate the performance of the FDDI systems based on the parameters namely throughput and response time. Results shown in the last section conclude that efficiency of the FDDI network can be increased by controlling the ring latency and by minimizing it. The response time can be improved by decreasing the transmission delay and propagation time. The transmission delay can be decreased by increasing the bandwidth and propagation time can be decreased by increasing the group velocity.

In this way we can evaluate the performance of the FDDI networks and can produce a FDDI network with greater efficiency and faster to operate

\section{REFERENCES}

[1] Raj Jain, "Performance analysis of FDDI Token Ring Network: Effect of parameters and guidelines for setting TTRT" Association for computing machinery, Inc. SIGCOMM’ 90, September 1990.

[2] Zhaoyi Lu, Lihong Wang, Dongyi Yao, "Study on the modelling of timed-token protocol", Applied Mathematical Modelling Vol. 26, pp. 797-805, (2002).
[3] Harry YUKLEA, "An FDDI Overview Some Question Answered" , Computer Networks and ISDN Systems Vol19, pp. 228-232, (1990).

[4] Izhak Rubin, James C.-H. Wu , "Analysis of an FDDI network supporting stations with single-packet buffers", Computer Networks and ISDN Systems, Vol 29, pp. 249266, (1997).

[5] Peter Domschitz and Martin Siegel, "Dual ring usage in FDDI", Computer communications, Vol 15, no 7 , september 1992.

[6] Qin Zheng, "Synchronous Bandwidth Allocation in FDDI Networks," Mitsubishi Electric Research Laboratories, TR93-16 December 1993

[7] R Radhakrishna Pillai, Anand Hardi and A Selvarajan, "Improving fault detection and reliability in FDDI networks", Computer Communications, Vol 15, No 9, pp. 587, November 1992

[8] Peter Davids, Thomas Meuser, Otto Spaniol, "FDDI: status and perspectives", Computer Networks and ISDN Systems, Vol. 26, pp. 657-677, (1994). 